\title{
Brain Findings Associated with Iodine Deficiency Identified by Magnetic Resonance Methods: A Systematic Review
}

\author{
Maria del C. Valdés Hernández ${ }^{1}$, Kirsty L. Wilson ${ }^{2}$, Emilie Combet ${ }^{3}$, Joanna M. Wardlaw ${ }^{1}$ \\ ${ }^{1}$ Brain Research Imaging Centre, University of Edinburgh, Edinburgh, UK \\ ${ }^{2}$ College of Medicine and Veterinary Medicine, University of Edinburgh, Edinburgh, UK \\ ${ }^{3}$ Department of Human Nutrition, University of Glasgow, Glasgow, UK \\ Email: mvhernan@staffmail.ed.ac.uk
}

Received July 15, 2013; revised August 15, 2013; accepted August 23, 2013

Copyright (C) 2013 Maria del C. Valdés Hernández et al. This is an open access article distributed under the Creative Commons Attribution License, which permits unrestricted use, distribution, and reproduction in any medium, provided the original work is properly cited. In accordance of the Creative Commons Attribution License all Copyrights (C 2013 are reserved for SCIRP and the owner of the intellectual property Maria del C. Valdés Hernández et al. All Copyright (C) 2013 are guarded by law and by SCIRP as a guardian.

\begin{abstract}
Objectives: Iodine deficiency (ID) is a common cause of preventable brain damage and mental retardation worldwide, according to the World Health Organisation. It may adversely affect brain maturation processes that potentially result in structural and metabolic brain abnormalities, visible on Magnetic Resonance (MR) techniques. Currently, however, there has been no review of the appearance of these brain changes on MR methods. Methods: A systematic review was conducted using 3 online search databases (Medline, Embase and Web of Knowledge) using multiple combinations of the following search terms: iodine, iodine deficiency, magnetic resonance, MRI, MRS, brain, imaging and iodine deficiency disorders (i.e. hypothyroxinaemia, congenital hypothyroidism, hypothyroidism and cretinism). Results: Up to May 2013, 1673 related papers were found. Of these, 29 studies confirmed their findings directly using MR Imaging and/or MR Spectroscopy. Of them, 28 were in humans and involved 157 subjects, 46 of whom had primary hypothyroidism, 97 had congenital hypothyroidism, 3 had endemic cretinism and 11 had subclinical hypothyroidism. The studies were small, with a mean relevant sample size of 6 , median 2 , range $1-35$, while 14 studies were individual case reports. T1-weighted was the most commonly used MRI sequence (20/29 studies) and 1.5 Tesla was the most commonly used magnet strength (6/10 studies that provided this information). Pituitary abnormalities (18/29 studies) and cerebellar atrophy (3/29 studies) were the most prevalent brain abnormalities found. Only fMRI studies (3/29) reported cognition-related abnormalities but the brain changes found were limited to a visual description in all studies. Conclusions: More studies that use MR methods to identify changes on brain volume or other global structural abnormalities and explain the mechanism of ID causing thyroid dysfunction and hence cognitive damage are required. Given the role of MR techniques in cognitive studies, this review provides a starting point for researching the macroscopic structural brain changes caused by ID.
\end{abstract}

Keywords: Iodine Deficiency; MRI; Brain; Hypothyroidism

\section{Introduction}

Iodine is an important micronutrient and a fundamental substrate for the synthesis of thyroid hormones [1,2]. Triiodothyronine (T3) and thyroxine (T4) are examples of iodinated thyroid hormones essential for several cellular metabolic processes and the development of the central nervous system [3]. Thyroid hormone functions are impaired by iodine deficiency [4], reflected as increased plasma thyroid stimulating hormone (TSH) and plasma T3 concentrations with reduced tissue and plasma T4 levels [5].

Iodine deficiency (ID) is one of the three key micronutrient deficiencies highlighted as major public health issues by the World Health Organisation: in 1990, 1.6 billion people, or $28.9 \%$ of the global population, were at risk and it was thus considered a serious public health issue throughout the world [6-8]. In 2011 this figure had 
risen to 2 billion and, after starvation, ID is currently the single greatest cause of preventable mental retardation and brain damage [9]. European countries are usually assumed to be iodine sufficient, however, several pockets of insufficiency have been described (including UK, Italy, Belgium), with no official data available for several countries.

The degree of neurological impairment and the likelihood of its permanence are not only related to the severity of ID but also to the stage of life at which the individual is exposed to it [10]. Iodine deficiency disorders, such as hypothyroidism, may reflect a maternal, fetal or neonatal childhood thyroid hormone insufficiency [11]. At differing time-points, thyroid hormones have particular effects on brain maturation, regulation of neuronal development and microglial proliferation [12], dendritic arborisation, synaptogenesis, cell migration and myelination [11]. Fetal's thyroid hormones rely on iodine supplemented from the maternal circulation so as to ensure adequate mental development [13]. Iodine insufficiency in fetal life and early childhood is associated with decreased IQ even in the absence of manifest hypothyroidism [14]. Many studies in areas of mild iodine deficiency have shown a range of developmental impairments including poor visual-motor performance and motor skills, decreased neuromotor and perceptual ability and lower developmental and intelligence quotients [7]. There is clearly an association between sub-optimal intellectual performance and iodine deficiency including maternal iodine deficiency during pregnancy [7,14-18].

Despite recommendations to increase daily iodine intake from $150 \mu \mathrm{g} / \mathrm{day}$ to $250 \mu \mathrm{g} /$ day during pregnancy, up to $40 \%$ of pregnant women in Scotland have been shown to be at risk of iodine deficiency [19]. Although few people have frank iodine deficiency and diet-driven hypothyroidism, a low or marginal intake will present a potential hazard in pregnancy, when demand is increased [20]. Iodine is obtained mainly through the diet. The iodine content of food and water is dependent on a variety of factors: including geographical location, mineral content of the soil, bacteria, rainfall, altitude and fertilisers used [21] as well as longstanding fortification programmes with iodine-supplemented salt introduced to counteract dietary deficiency $[22,23]$. There is no ongoing iodine-fortification programme in the UK [24]. Main sources of iodine in the British diet are milk and dairy products, and fish and seafood [25]. It is likely that a substantial proportion of the young female population excludes at least one of these food groups from their diets, leading to either low or marginal iodine intake [16]. Meanwhile, fast-food meals and pre-cooked dishes do not ensure that the minimum iodine requirement is fulfilled: 150 - $300 \mu \mathrm{g}$ I per day [26]. The most recent survey conducted in the UK revealed a median urinary io- dine excretion (i.e. marker of ID at population level) of $80 \mu \mathrm{g} / \mathrm{L}$, indicative of mild deficiency (50 - $99 \mu \mathrm{g} / \mathrm{L})$ [9].

The brain is particularly sensitive to the adverse effects of ID since neural development occurs at a critical period, prior to the rest of the body [27]. This is reflected in the disproportionate weight of the brain in a neonate, representing $10 \%$ of total body mass, compared to $2 \%$ in a fully grown adult [27]. Animal models of ID have provided evidence of changes to the morphology and cytoarchitecture of the brain. In sheep models of ID, reduced brain DNA and brain weight with delayed cerebellar maturation were identified [28-30]. In rat brains studies have reported altered metabolic activity and laminar volumes in the hippocampus and dentate gyrus [31] and altered tissue distribution of other trace elements [32-34]. It is also suggested that certain brain proteins may be down-regulated in particular brain regions [35], anterior commissure axons and mRNA expression may be reduced [36,37], dendrite size may be altered [38] and premature cell apoptosis may result [39]. Additionally, ID may cause a reduction in cerebellar cell size and decreased myelination throughout the Central Nervous System [40].

Magnetic Resonance (MR) is a powerful, non-invasive tool for detecting and quantifying structural and metabolic brain changes in life over time. Although access is limited in some regions, it is increasingly available throughout the world. Despite the strong link between iodine insufficiency and neurodevelopment and impaired cognition, brain structural changes have rarely been investigated in the context of iodine insufficiency. We hypothesise that insufficient dietary iodine intake or aberrant iodine metabolism results in structural and metabolic neurological changes in the brain that can be assessed by MR methods. Currently, few reports exist regarding the appearance of these changes on MR. Studies in ID disorders report histological, psychological, physical and behavioural changes but use no brain MR confirmation and brain changes are often inconsistently described. This systematic review was necessary to clarify reported changes on brain MR.

\section{Aims and Hypothesis}

\subsection{Aims}

This review aims to identify what brain structural and metabolic abnormalities related to ID are documented using Magnetic Resonance Imaging (MRI) and other MR techniques such as Magnetic Resonance Spectroscopy (MRS).

\subsection{Hypothesis}

The hypothesis of this review is that insufficient dietary 
iodine content or aberrant iodine metabolism results in structural and metabolic neurological changes in the brain, detectable on MR methods.

\section{Methods}

\subsection{Search Criteria}

Primary research studies, published in full and using MR techniques to determine brain region modifications, were identified in a literature search. A combination of case reports, prospective and retrospective studies were reviewed. The electronic search was conducted up to May 2013 using the following databases: Medline ${ }^{\mathcal{O}}$, Web of Knowledge $^{\odot}$ and Embase $^{\odot}$. It was supplemented by hand-searching reference lists of the review papers and by request to the corresponding authors of identified papers not openly accessible. Multiple combinations of the following search terms were used: iodine, iodine deficiency, iodine deposits, magnetic resonance imaging/ MRI, brain, imaging, hypothyroxinaemia, congenital hypothyroidism, hypothyroidism and cretinism. The latter four search terms were proposed as they may be considered as iodine deficiency disorders. Maternal hypothyroxinaemia may result from inadequate iodine intake [41-44] and may cause neurodevelopmental defects. Neonatal hypothyroxinaemia, from postnatal reductions in T4 concentration, but with normal TSH, may occur due to in utero iodine insufficiencies $[45,46]$. Congenital hypothyroidism, caused by maternal and thence fetal hypothyroidism, may, therefore, also result from iodine deficiency, with an incidence of 1:3000 to 1:4000 live births $[47,48]$. One of the worst consequences of ID and a more severe form of hypothyroidism is endemic cretinism, a condition characterised by neurological deficits, deaf-mutism and spasticity [49-51] that occurs where ID is common in the community.

One reviewer independently carried out the primary literature search, paper selection, duplicate removal and data extraction up to March 2012 and other reviewer extended the search up to May 2013. Three different reviewers assessed a sample of papers for inclusion and helped extract the relevant data on each occasion. Although papers may have passed eligibility checks according to the inclusion/exclusion criteria listed below, full texts were read prior to final rejection of studies.

\subsection{Inclusion Criteria}

Studies were included which used MR methods to identify brain structural and/or metabolic changes in the brain associated with ID or ID disorders. Inclusion criteria also comprised studies available in English only. Both human and animal studies were included as well as studies from healthy or diseased brains.

\subsection{Exclusion Criteria}

Studies were excluded if they did not meet the inclusion criteria or were published only as abstracts without full publication available. Studies in which iodine was used therapeutically or as a drug treatment were rejected and studies which involved the injection of radioactive iodine used as a contrast agent for visualising a specific pathology (e.g. thyroid carcinoma) were also excluded. Studies in which hypothyroidism was induced by thyroidectomy or was due to other non-iodine related causes (such as autosomal, goitrogen, steroid consumption, following head trauma, caused by Hashimoto's thyroiditis, stress or autoimmune aetiology) were rejected. Studies on cancer patients or those in which the MR method did not involve studying or imaging the brain (e.g. thyroid scintigraphy) were also rejected. The literature search produced many papers which involved non relevant subject areas and/or diseases; diabetes mellitus, multiple sclerosis, epilepsy, Parkinson's, Alzheimer's, bipolar disorder and Turner's syndrome are examples which were excluded as the non-iodine related consequences of these diseases may affect the appearance of brain changes. Reviews which discuss MR changes associated with iodine deficiency were excluded from the data analysis unless they included new data that was not published elsewhere. If the cause of the disorder (e.g. hypothyroidism) was not ID or not stated the study was rejected.

\subsection{Data Extraction}

For each study that was included: the type of MR confirmation, the appearance on MR method, the location of the abnormality, the pathology/disease studied and the sample size (i.e. number of subjects) was independently extracted. Often the particular type of MR technique used was not discussed in the body of the text and so the relevant information was extracted from MR image descriptions. Additionally, information related to subject pathology was sometimes acquired from tables.

\subsection{Data Analysis}

The techniques used to identify structural and/or metabolic brain changes were quantified, including:

Number of subjects included in the studies

- How many studies successfully used MR techniques to determine brain changes

- How many studies that used MR methods reported discrepancies on the brain changes

- Whether the studies included blinding, randomisation or an inclusion/exclusion criteria

Moreover, two further questions were posed:

- Where are the most common locations of the brain changes? 
- What diseases/pathologies discussed in the studies were associated with which brain changes/ MR abnormality appearances?

However, these last two questions are not the main focus of this review since results only encompass studies which used MR confirmation, rather than all of the literature that discusses the relationship between iodine deficiency and the brain.

\section{Results}

The literature search identified 1673 publications; 553 from Medline, 625 from Web of Knowledge, 490 from Embase and 5 from review paper reference lists. 29 of these studies were included in the review (Table 1 and
Figure 1). 1155 papers were rejected because the paper involved non-relevant diseases [Exclusion Criteria], was not related to the effects of ID on the brain and/or used iodine as a contrast medium or therapy, the full paper was not written in English (i.e. no translation was available for 330 papers) or was not attainable through the search databases. Further 60 duplicate papers were rejected. Of the remaining 128, 46 review articles and 47 that did not confirm findings using MR techniques (i.e. used CT, Nuclear Medicine methods and/or ultrasound) or those in which the MR technique was not applied to the brain, such as thyroid or whole body scintigraphy, were excluded from the data analysis. Finally, six studies on hypothyroidism that did not specify the cause [Exclu-

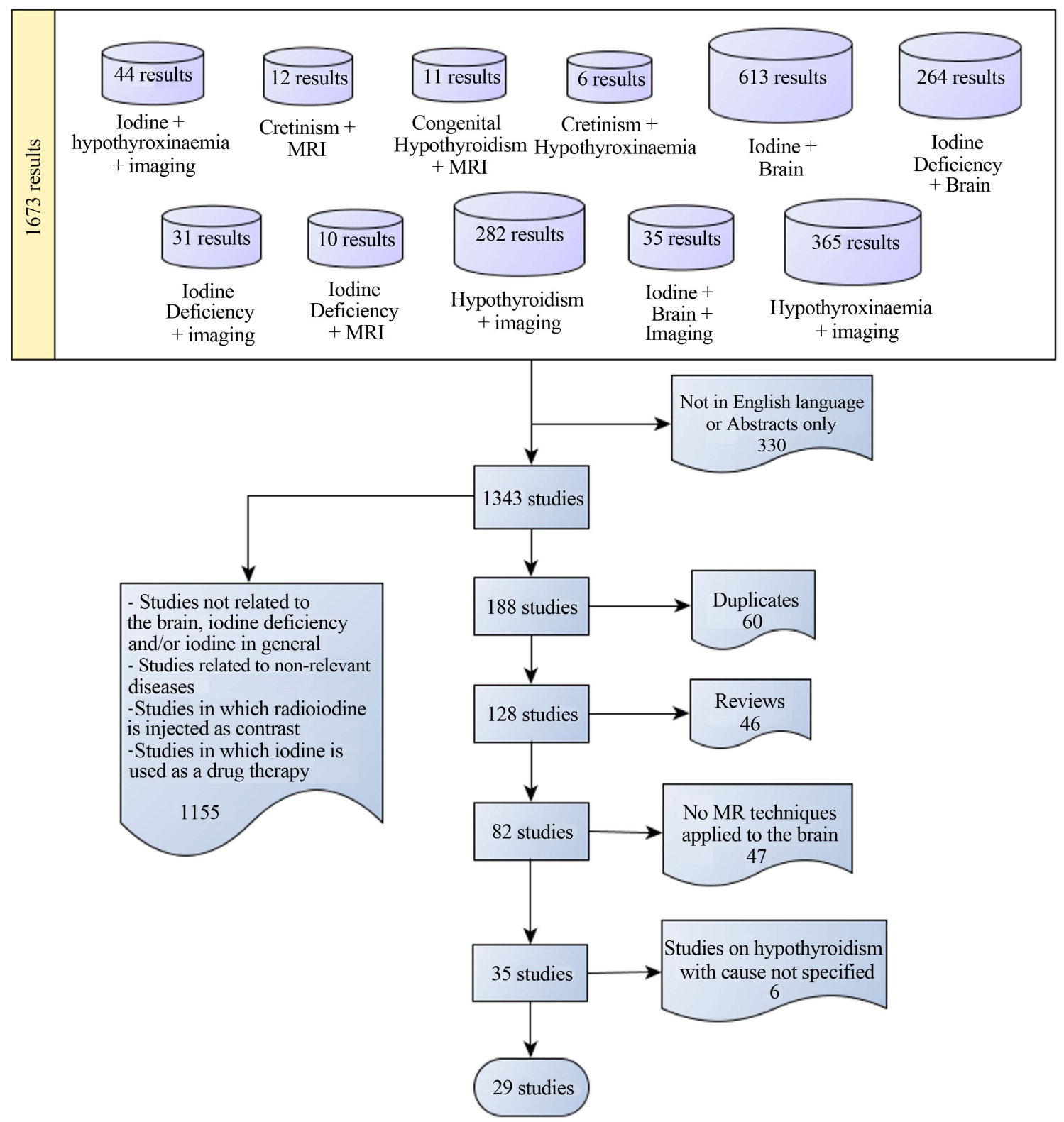

Figure 1. Flow chart summarising the spectrum of results from the literature search. 
Table 1. Summary of included studies (Agrawal 2011 to Zhu 2006).

\begin{tabular}{|c|c|c|c|c|c|c|c|c|}
\hline Reference & $\begin{array}{l}\text { Pathology } \\
\text { studied }\end{array}$ & $\begin{array}{c}\text { Sample } \\
\text { type }\end{array}$ & $\begin{array}{l}\text { Relevant } \\
\text { Sample size }\end{array}$ & $\begin{array}{c}\text { MR } \\
\text { method }\end{array}$ & $\begin{array}{l}\text { MR sequence } \\
\text { /modality }\end{array}$ & $\begin{array}{c}\text { Finding/appearance } \\
\text { on MR }\end{array}$ & $\begin{array}{c}\text { Abnormality } \\
\text { location }\end{array}$ & $\begin{array}{l}\text { Magnetic } \\
\text { field } \\
\text { strength }\end{array}$ \\
\hline Agrawal $2011^{66}$ & PH & Human & 1 & MRI & $\mathrm{T} 1 \mathrm{~W}+\mathrm{T} 2 \mathrm{~W}$ & Enlargement & Pituitary & l \\
\hline Aijing $2010^{52}$ & PH & Human & 8 & MRI & / & Enlargement & Pituitary & I \\
\hline Akinci $2006^{67}$ & $\mathrm{CH}$ & Human & 8 & MRS + MRI & $\mathrm{T} 1 \mathrm{~W}+\mathrm{T} 2 \mathrm{~W}$ & Decreased NAA/Cr ratios & $\begin{array}{c}\text { PWM + } \\
\text { Thalamus }\end{array}$ & $1.5 \mathrm{~T}$ \\
\hline Alves $1989^{68}$ & $\mathrm{CH}$ & Human & 1 & MRI & $\begin{array}{l}\mathrm{T} 2 \mathrm{~W} \text { (spin echo and } \\
\text { inversion recovery) }\end{array}$ & $\begin{array}{l}\text { Patchy hyperintensities } \\
\text { in white matter }\end{array}$ & $\begin{array}{l}\text { Splenium and } \\
\text { Frontal lobe }\end{array}$ & $0.5 \mathrm{~T}$ \\
\hline Ashley $2005^{53}$ & $\mathrm{PH}$ & Human & 1 & MRI & $\mathrm{T} 1 \mathrm{~W}$ & Enlargement & Pituitary & / \\
\hline Atchison $1989^{65}$ & $\mathrm{PH}$ & Human & 3 & MRI & $\begin{array}{l}\text { /(images show } \mathrm{T} 1 \mathrm{~W} \\
\text { seq) }\end{array}$ & Enlargement & $\begin{array}{l}\text { Pituitary } \\
\text { (sellar and } \\
\text { suprasellar) }\end{array}$ & / \\
\hline Blasi $2009^{69}$ & $\mathrm{CH}$ & Human & 15 & $\mathrm{fMRI}+\mathrm{MRI}$ & $\mathrm{T} 1 \mathrm{~W}+\mathrm{T} 2 \mathrm{~W}$ & $\begin{array}{l}\text { Decreased sup. + inf. parietal } \\
\text { cortex activation; increased } \\
\text { SMA, precentral gyrus, } \\
\text { insula + L somatosensory } \\
\text { parietal cortex activation }\end{array}$ & $\begin{array}{l}\text { Parietal cortex, } \\
\text { SMA, } \\
\text { precentral } \\
\text { gyrus, insula }\end{array}$ & $1.5 \mathrm{~T}$ \\
\hline Dedov $1994^{54}$ & $\mathrm{PH}$ & Human & 10 & MRI & Spin-echo & $\begin{array}{l}\text { Empty sella turcica } \\
\text { (on 6/10 subjects) }\end{array}$ & Pituitary & $0.234 \mathrm{~T}$ \\
\hline Desai $1996^{70}$ & $\mathrm{CH}$ & Human & 10 & MRI & $\begin{array}{l}\mathrm{T} 1 \mathrm{~W}+\mathrm{T} 2 * \mathrm{~W} \\
\text { (gradient echo) on } \\
\text { selected patients }\end{array}$ & Enlargement & Pituitary & $0.2 \mathrm{~T}$ \\
\hline Dutta $2012^{64}$ & $\mathrm{PH}$ & Human & 1 & MRI & $\mathrm{T} 1 \mathrm{~W}$ & Empty sella turcica & Pituitary & l \\
\hline Ehirim $1998^{55}$ & $\mathrm{PH}$ & Human & 1 & MRI & $\mathrm{T} 1 \mathrm{~W}$ & Enlargement & Pituitary & / \\
\hline $\begin{array}{l}\text { Garcia-Centeno } \\
2010^{56}\end{array}$ & $\mathrm{PH}$ & Human & 13 & MRI & $\mathrm{T} 1 \mathrm{~W}$ & Empty sella turcica & Pituitary & $1.5 \mathrm{~T}$ \\
\hline Goswami $1999^{57}$ & $\mathrm{PH}$ & Human & 1 & MRI & $\mathrm{T} 1 \mathrm{~W}$ & Enlargement & Pituitary & / \\
\hline Graber $2009^{71}$ & $\mathrm{CH}$ & Human & 1 & MRI & $\mathrm{T} 1 \mathrm{~W}$ & Hypoplasia & $\begin{array}{l}\text { Cerebellar } \\
\text { vermis }\end{array}$ & / \\
\hline Gupta $2005^{72}$ & $\mathrm{CH}$ & Human & 5 & $\begin{array}{c}\text { MRS + } \\
\text { MRI }\end{array}$ & $\begin{array}{c}\text { STEM, } \\
\text { T1W + T2W }\end{array}$ & $\begin{array}{l}\text { Increased Cho/Cr ratio } \\
\text { Mild cerebral cortical } \\
\text { atrophy in frontal } \\
\text { and parietal lobes }\end{array}$ & l & $2 \mathrm{~T}$ \\
\hline Hasegawa $2010^{80}$ & PerH & Rat & 8 & MRI & I & $\begin{array}{l}\text { Reduced total brain volume } \\
\text { but ratio of hippocampal } \\
\text { volume to total brain volume } \\
\text { remains constant }\end{array}$ & $\begin{array}{l}\text { Brain as a } \\
\text { whole }\end{array}$ & $7 \mathrm{~T}$ \\
\hline Kroese $2004^{58}$ & $\mathrm{PH}$ & Human & 1 & MRI & / & Enlargement & Pituitary & / \\
\hline Lee $2008^{59}$ & $\mathrm{PH}$ & Human & 1 & MRI & $\mathrm{T} 1 \mathrm{~W}+\mathrm{T} 2 \mathrm{~W}$ & Enlargement & Pituitary & / \\
\hline Mauceri $1997^{73}$ & $\mathrm{CH}$ & Human & 1 & MRI & $\mathrm{T} 2 \mathrm{~W}$ & Hypoplasia & $\begin{array}{l}\text { R. cerebellar } \\
\text { vermis \& } \\
\text { hemisphere }\end{array}$ & / \\
\hline Fujiwara $2008^{74}$ & $\mathrm{CH}$ & Human & 6 & MRI & I & $\begin{array}{l}\text { Decreased anterior pituitary, } \\
\text { ectopic posterior pituitary } \\
\quad \text { (on } 4 / 6 \text { subjects) }\end{array}$ & Pituitary & / \\
\hline Passeri $2011^{60}$ & $\mathrm{PH}$ & Human & 1 & MRI & $\mathrm{T} 1 \mathrm{~W}$ & Enlargement & Pituitary & / \\
\hline Sengupta $2012^{63}$ & $\mathrm{PH}$ & Human & 1 & MRI & $\mathrm{T} 1 \mathrm{~W}$ & Enlargement (hyperplasia) & Pituitary & / \\
\hline Shogan $2010^{61}$ & $\mathrm{PH}$ & Human & 1 & MRI & $\mathrm{T} 1 \mathrm{~W}$ & $\begin{array}{l}\text { Sella turcica expansion with } \\
\text { pituitary enlargement }\end{array}$ & Pituitary & / \\
\hline Tai $1993^{78}$ & $\mathrm{EC}$ & Human & 3 & MRI & $\mathrm{T} 1 \mathrm{~W}+\mathrm{T} 2 \mathrm{~W}$ & Altered intensity & $\begin{array}{l}\text { Globus Pallidus } \\
+ \text { Substantia } \\
\text { Nigra }\end{array}$ & / \\
\hline
\end{tabular}


Continued

\begin{tabular}{ccccccccc}
\hline Tajima $2007^{75}$ & CH & Human & 1 & MRI & $/$ & Atrophy & Cerebellum \\
Wheeler $2011^{76}$ & $\mathrm{CH}$ & Human & 35 & MRI & T1W + T2W & $\begin{array}{c}\text { Decreased L. } \\
\text { hippocampal size }\end{array}$ & Hippocampus & $1.5 \mathrm{~T}$ \\
Wheeler $2012^{77}$ & $\mathrm{CH}$ & Human & 14 & fMRI & T1W & Increased bilateral activation Hippocampus & $1.5 \mathrm{~T}$ \\
Young $1999^{62}$ & PH & Human & 2 & MRI & $/$ & Enlargement & Pituitary \\
Zhu $2006^{79}$ & SH & Human & 11 & fMRI + MRI & T1W + T2W & Decreased activation & IFG, DLPFC + SMA & $1.5 \mathrm{~T}$ \\
\hline
\end{tabular}

sion Criteria] were excluded from the main data analysis and discussed separately.

\subsection{Sample Size}

In total, 165 subjects were included from the 29 studies. Of these, 46 subjects were in studies on primary hypothyroidism [52-66] (i.e. in which hypothyroidism was related to ID), 97 subjects in studies on congenital hypothyroidism [67-77], 3 subjects in a study of endemic cretinism [78], 11 subjects in a study of subclinical hypothyroidism $[79,80]$ and 8 subjects in a study of perinatal hypothyroidism [80] (Figure 2). One study used animals [80], involving 8 congenital hypothyroid and 8 control rats aged between 7 and 11 months old, yet there was no reporting of death numbers or inclusion/exclusion criteria. The mean sample size (for subjects included in all studies) was 6 , the median was 2 and the range $1-35$, but 14 studies were single case reports [53,55,57-61,63, $64,66,68,71,73,75]$. The age range of the human subjects was 0 - 50 years. The study with the largest number of included subjects was from Wheeler et al. (2011) [76], with 35 congenital hypothyroid patients.

\subsection{Blinding, Randomisation and Inclusion/Exclusion Criteria}

The quality of the data presented by this study was assessed following the PRISMA statement [81] and a modification of the QUADAS tool [82]. Results are shown in Table 2. Two studies used blinding of assessors to iodine status $[69,76]$, and the image processing on other study [77] was done fully automatically. Although the data processing in all fMRI studies was also done fully automatically, there was no evidence of blindness in the imaging data acquisition. Randomisation was only provided in the animal study whereby rats were randomly assorted into control and treatment groups for MRI experimentation [80]. Ten studies gave implicit inclusion/exclusion criteria through the description of the characteristics of the sample and five gave them explicitly $[67,69,74,76,77]$. Reasons for rejection were patients with unrelated disorders which may affect cerebral function or development such as chromosomal or metabolic
Number of included subjects with associated diseases/pathologies

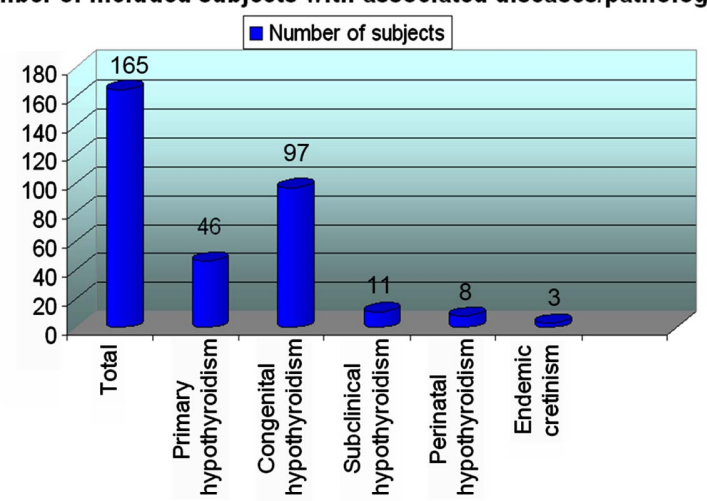

Figure 2. Number of subjects on each pathology included.

disorders, risk factors for brain injury (e.g. hypoxia, teratogens), central nervous system disorders, head trauma, prematurity, congenital malformations or a psychiatric disorder.

\subsection{Type of MR Confirmation}

The most commonly used imaging method was structural MRI (29/29 studies) (Tables 1 and 3), predominantly with conventional structural sequences. MRS was used in 2 studies $[67,72]$ and functional MRI (fMRI) in 3 studies $[69,77,79]$. T1-weighted (T1W) was the sequence most widely used (in 20/29 studies). T2-weighted (T2W) sequence was utilised in 11/29 studies whilst 6/29 studies did not clarify the MRI sequence used. In 2 studies, only $60 \%$ of the hypothyroid patients showed brain changes on MRI [54,74] (Table 3).

Only 7 MRI studies utilised contrast enhancement; Gadolinium was used in each of these [55-57,60-62,65]. Both MRS studies [67,72] used shimming techniques and water suppression chemical shift selection pulses but only one study discussed the additional use of Stimulated Echo Acquisition Mode (STEAM) [72].

None of the 29 included papers investigated the effect of using different MR magnetic field strengths on the appearance of brain changes caused by ID, with 11/29 of the studies not clarifying the strength used. Most studies used a 1.5 Tesla magnet strength (6/29 studies) with two using a low 0.2 Tesla strength $[54,70]$. The animal study 
Table 2. Study quality and risk of bias.

\begin{tabular}{|c|c|c|c|c|c|c|c|c|c|c|}
\hline Referen-ce & $\begin{array}{l}\text { Patients } \\
\text { blinded }\end{array}$ & $\begin{array}{l}\text { Data } \\
\text { collectors } \\
\text { blinded }\end{array}$ & $\begin{array}{l}\text { Outcome } \\
\text { assesors } \\
\text { blinded }\end{array}$ & $\begin{array}{l}\text { Gender } \\
\text { balance }\end{array}$ & $\begin{array}{ll} & \text { Random } \\
\text { e selection }\end{array}$ & $\begin{array}{l}\text { Sample } \\
\text { representative } \\
\text { according to the } \\
\text { aim of the study }\end{array}$ & $\begin{array}{c}\text { Tests are } \\
\text { repeatable }\end{array}$ & $\begin{array}{l}\text { Results } \\
\text { can be } \\
\text { replicated }\end{array}$ & $\begin{array}{l}\text { Withdrawals } \\
\text { from the } \\
\text { study } \\
\text { explained }\end{array}$ & $\begin{array}{l}\text { Inclusion/ } \\
\text { exclusion criteria } \\
\text { given }\end{array}$ \\
\hline $\begin{array}{l}\text { Agrawal } \\
2011^{66}\end{array}$ & & & & $1 \mathrm{~F}$ & $\mathrm{n} / \mathrm{a}$ & $\checkmark$ & & & $\mathrm{n} / \mathrm{a}$ & $\begin{array}{c}\mathrm{n} / \mathrm{a} \\
\text { (case report) }\end{array}$ \\
\hline Aijing $2010^{52}$ & $\begin{array}{c}\checkmark \\
\text { (retrospective) }\end{array}$ & & & $\mathrm{n} / \mathrm{s}$ & $\begin{array}{l}\text { all cases } \\
\text { presented } \\
\text { on a centre }\end{array}$ & $\checkmark$ & $\checkmark$ & & $\mathrm{n} / \mathrm{a}$ & $\checkmark$ \\
\hline Akinci $2006^{67}$ & & & & $\mathrm{n} / \mathrm{s}$ & $\mathrm{n} / \mathrm{s}$ & $\checkmark$ & $\checkmark$ & $\checkmark$ & $\mathrm{n} / \mathrm{a}$ & $\checkmark$ \\
\hline Alves $1989^{68}$ & & & & $1 \mathrm{~F}$ & $\mathrm{n} / \mathrm{a}$ & & $\checkmark$ & & $\mathrm{n} / \mathrm{a}$ & $\begin{array}{c}\mathrm{n} / \mathrm{a} \\
\text { (case report) }\end{array}$ \\
\hline Ashley $2005^{53}$ & & & & $1 \mathrm{~F}$ & $\mathrm{n} / \mathrm{a}$ & & $\checkmark$ & & $\mathrm{n} / \mathrm{a}$ & $\begin{array}{c}\mathrm{n} / \mathrm{a} \\
\text { (case report) }\end{array}$ \\
\hline $\begin{array}{l}\text { Atchison } \\
1989^{65}\end{array}$ & & & & $\begin{array}{l}2 \mathrm{~F} \\
1 \mathrm{M}\end{array}$ & $\mathrm{n} / \mathrm{s}$ & $\checkmark$ & $\checkmark$ & & $\mathrm{n} / \mathrm{a}$ & $\begin{array}{l}\text { (inclusion only) } \\
3 \text { case reports }\end{array}$ \\
\hline Blasi $2009^{69}$ & & & $\checkmark$ & $\begin{array}{l}10 \mathrm{~F} \\
5 \mathrm{M}\end{array}$ & $\begin{array}{l}\text { all (those } \\
\text { consented) } \\
\text { identified } \\
\text { by neonatal } \\
\text { screening }\end{array}$ & 1 & $\checkmark$ & $\checkmark$ & $\mathrm{n} / \mathrm{a}$ & $\checkmark$ \\
\hline Dedov $1994^{54}$ & $\begin{array}{c}\checkmark \\
\text { (retrospective) }\end{array}$ & & & $\mathrm{n} / \mathrm{s}$ & $\begin{array}{r}\text { Included } \\
\text { patients (1 } \\
\text { inclusi }\end{array}$ & $\begin{array}{l}\text { all presented } \\
110 \text { ), given the } \\
\text { ion criteria }\end{array}$ & $\checkmark$ & & $\mathrm{n} / \mathrm{a}$ & $\checkmark$ \\
\hline Desai $1996^{70}$ & & & & $\begin{array}{l}5 \mathrm{~F} \\
5 \mathrm{M}\end{array}$ & $\mathrm{n} / \mathrm{s}$ & & $\checkmark$ & & $\mathrm{n} / \mathrm{s}$ & \\
\hline Dutta $2012^{64}$ & & & & $1 \mathrm{M}$ & & & & & $\mathrm{n} / \mathrm{a}$ & $\mathrm{n} / \mathrm{a}$ (case report) \\
\hline Ehirim $1998^{55}$ & & & & $1 \mathrm{M}$ & & $\checkmark$ & & & $\mathrm{n} / \mathrm{a}$ & $\mathrm{n} / \mathrm{a}$ (case report) \\
\hline $\begin{array}{l}\text { Garcia } \\
\text { Centeno } \\
2010^{56}\end{array}$ & $\begin{array}{c}\checkmark \\
\text { (retrospective) }\end{array}$ & & & $\begin{array}{l}2 \mathrm{M} \\
11 \mathrm{~F}\end{array}$ & $\begin{array}{r}\text { Included } \\
\text { patients }( \\
\text { inclusi }\end{array}$ & $\begin{array}{l}\text { all presented } \\
(56) \text {, given the } \\
\text { sion criteria }\end{array}$ & $\checkmark$ & $\mathrm{n} / \mathrm{a}$ & $\mathrm{n} / \mathrm{a}$ & $\checkmark$ \\
\hline $\begin{array}{c}\text { Goswami } \\
1999^{57}\end{array}$ & & & & $1 \mathrm{M}$ & & & $\checkmark$ & & $\mathrm{n} / \mathrm{a}$ & $\mathrm{n} / \mathrm{a}$ (case report) \\
\hline Graber $2009^{71}$ & & & & $1 \mathrm{~F}$ & & & $\checkmark$ & & $\mathrm{n} / \mathrm{a}$ & $\mathrm{n} / \mathrm{a}$ (case report) \\
\hline Gupta $2005^{72}$ & & & & $\mathrm{n} / \mathrm{s}$ & & & $\checkmark$ & & & $\mathrm{n} / \mathrm{s}$ \\
\hline $\begin{array}{c}\text { Hasegawa } \\
2010^{81}\end{array}$ & $\checkmark$ & $\checkmark$ & $\checkmark$ & $\begin{array}{l}\text { Equal } \\
\mathrm{M} \text { and } \\
\mathrm{F}\end{array}$ & $\checkmark$ & & $\checkmark$ & $\checkmark$ & & $\checkmark$ \\
\hline Kroese $2004^{58}$ & & & & $1 \mathrm{~F}$ & $\mathrm{n} / \mathrm{a}$ & & $\begin{array}{c}\text { Conventional } \\
\text { exam }\end{array}$ & & $\mathrm{n} / \mathrm{a}$ & $\mathrm{n} / \mathrm{a}$ (case report) \\
\hline Lee $2008^{59}$ & & & & $1 \mathrm{~F}$ & $\mathrm{n} / \mathrm{a}$ & & $\begin{array}{c}\text { Conventional } \\
\text { exam. }\end{array}$ & & $\mathrm{n} / \mathrm{a}$ & $\mathrm{n} / \mathrm{a}$ (case report) \\
\hline $\begin{array}{c}\text { Mauceri } \\
1997^{73}\end{array}$ & & & & $1 \mathrm{M}$ & $\mathrm{n} / \mathrm{a}$ & $\checkmark$ & $\begin{array}{c}\text { Conventional } \\
\text { exam. }\end{array}$ & & $\mathrm{n} / \mathrm{a}$ & $\mathrm{n} / \mathrm{a}$ (case report) \\
\hline $\begin{array}{c}\text { Fujiwara } \\
2008^{74}\end{array}$ & & & & $\begin{array}{l}3 \mathrm{M} \\
3 \mathrm{~F}\end{array}$ & $\begin{array}{c}\text { Screened } \\
\text { all patients } \\
\text { born } \\
2000-2004\end{array}$ & $\checkmark$ & $\checkmark$ & & $\mathrm{n} / \mathrm{a}$ & $\checkmark$ \\
\hline $\begin{array}{l}\text { Passeri } \\
2011^{60}\end{array}$ & & & & $1 \mathrm{~F}$ & & $\checkmark$ & $\begin{array}{l}\text { Conventional } \\
\text { exam. }\end{array}$ & & $\mathrm{n} / \mathrm{a}$ & $\begin{array}{c}\mathrm{n} / \mathrm{a} \\
\text { (case report) }\end{array}$ \\
\hline $\begin{array}{l}\text { Sengupta } \\
2012^{63}\end{array}$ & & & & $1 \mathrm{~F}$ & & $\checkmark$ & $\checkmark$ & & $\mathrm{n} / \mathrm{a}$ & $\begin{array}{c}\mathrm{n} / \mathrm{a} \\
\text { (case report) }\end{array}$ \\
\hline
\end{tabular}




\begin{tabular}{|c|c|c|c|c|c|c|c|}
\hline \multicolumn{8}{|l|}{ Continued } \\
\hline Shogan $2010^{61}$ & & $1 \mathrm{~F}$ & & & & $\mathrm{n} / \mathrm{a}$ & $\begin{array}{c}\mathrm{n} / \mathrm{a} \\
\text { (case report) }\end{array}$ \\
\hline Tai $1993^{78}$ & & $3 \mathrm{M}$ & & & & $\mathrm{n} / \mathrm{a}$ & $\begin{array}{c}\mathrm{n} / \mathrm{a} \\
\text { (case report) }\end{array}$ \\
\hline Tajima $2007^{75}$ & & $1 \mathrm{M}$ & & $\checkmark$ & $\begin{array}{l}\text { Conventional } \\
\text { exam. }\end{array}$ & $\mathrm{n} / \mathrm{a}$ & $\begin{array}{c}\mathrm{n} / \mathrm{a} \\
\text { (case report) }\end{array}$ \\
\hline $\begin{array}{l}\text { Wheeler } \\
2011^{76}\end{array}$ & $\checkmark$ & $\begin{array}{l}14 \mathrm{M} \\
21 \mathrm{~F}\end{array}$ & & $\checkmark$ & $\checkmark$ & $\mathrm{n} / \mathrm{a}$ & $\checkmark$ \\
\hline $\begin{array}{l}\text { Wheeler } \\
2011^{77}\end{array}$ & $\begin{array}{l}\text { Computational } \\
\text { asessment }\end{array}$ & $\mathrm{n} / \mathrm{s}$ & & $\checkmark$ & $\checkmark$ & $\mathrm{n} / \mathrm{a}$ & $\checkmark$ \\
\hline Young $1999^{62}$ & & $3 \mathrm{~F}$ & & $\checkmark$ & $\begin{array}{l}\text { Conventional } \\
\text { exam. }\end{array}$ & $\mathrm{n} / \mathrm{a}$ & $\begin{array}{c}\mathrm{n} / \mathrm{a} \\
\text { (case report) }\end{array}$ \\
\hline Zhu $2006^{79}$ & & $\begin{array}{l}10 \mathrm{~F} \\
1 \mathrm{M}\end{array}$ & $\mathrm{n} / \mathrm{s}$ & & $\checkmark$ & $4 \mathrm{n} / \mathrm{s}$ & $\begin{array}{l}\text { Inclusion criteria } \\
\text { only }\end{array}$ \\
\hline
\end{tabular}

used a high strength 7 Tesla magnet [80]. Other magnet strengths were used by 1 study each: 0.5 Tesla [68] and 2 Tesla [72].

\subsection{Pathologies Associated with Brain Abnormalities}

Several studies of ID investigated brain changes in the context of a particular disease, with 27/29 studying changes in pathological brains. In the animal study, transient perinatal hypothyroidism was induced by anti-thyroid drug methimazole and so, although mimicking the consequences of ID, the rat brains were considered as non-diseased [80]. Zhu et al. [79] reported subjects with subclinical hypothyroidism and so the brains of these individuals were considered non-pathological since they did not have the overt form of the disease. Brain changes were seen on MR in all studies in the context of certain pathologies: primary, non-autoimmune hypothyroidism (15/29 studies), congenital hypothyroidism (11/29 studies), perinatal hypothyroidism (1/29 studies), endemic cretinism (1/29 study), subclinical hypothyroidism (1/29 studies) (Figure 2).

Primary hypothyroidism was most commonly associated with pituitary enlargement/hyperplasia (13/15 studies) whilst congenital hypothyroidism was related most strongly with cerebellar atrophy/hypoplasia (Figure 3 and Table 3).

\subsection{Location of the Abnormalities}

Thirteen out of 29 studies (32 subjects) reported homogenous diffuse enlargement of the pituitary gland and/or pituitary hyperplasia $[52,53,55,57-63,65,66,70]$. Several (7/29) also described suprasellar extension with compression of the optic chiasm; both features which mimic those of a suprasellar tumour. However, 4 other studies discussed empty sella turcica syndrome (i.e. absence of the diaphragm sella, reduced hypophysis volume and extension of the intrasellar subarachnoid region) $[54,56$,
Brain changes most commonly associated with particular diseases / pathologies
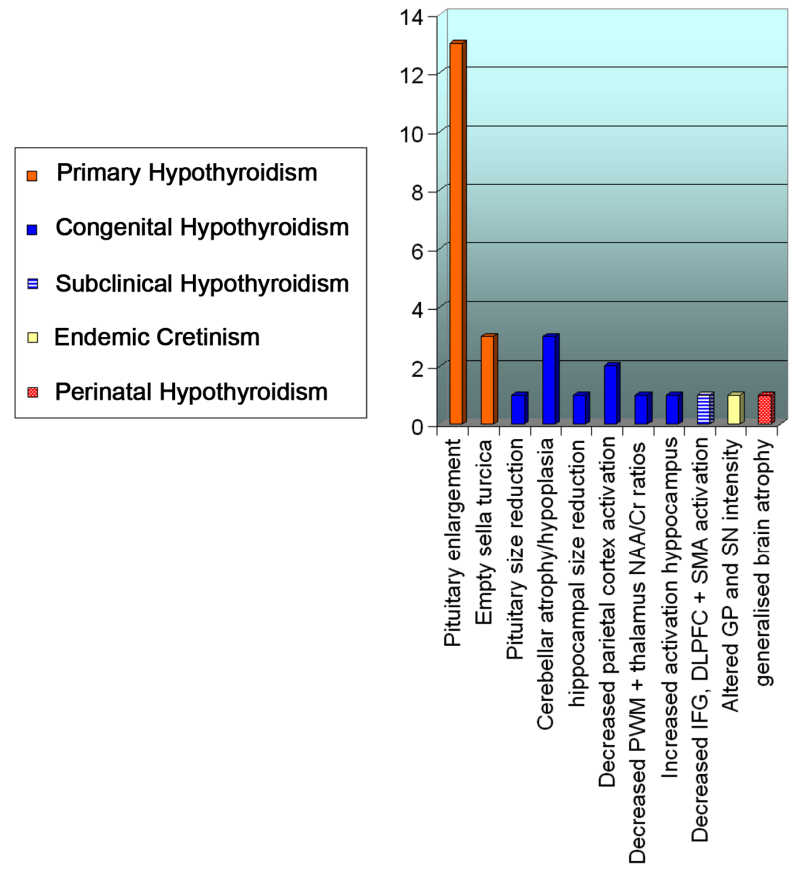

Figure 3. Studied imaged brain abnormalities in the context of a particular disease/pathology. This bar chart shows the brain abnormalities most commonly associated with particular disease/pathologies (caused by iodine deficiency) for the included studies.

64] and a decrease in anterior pituitary size combined with an ectopic posterior pituitary [74].

After the pituitary gland, the cerebellum was the next most cited location for brain abnormalities (Table 3) on 3 case studies of congenital hypothyroid subjects [71,73, 75], which reported abnormal cerebellar structure [71] and hypoplasia [71,73] or atrophy [75]. These findings suggest ID may contribute to aberrant development of the cerebellar hemispheres and vermis [73] or be associated with mechanisms that lead to regional atrophy $[71,75]$. 
Table 3. Appearance of brain abnormalities in the context of specified diseases, on the MRI methods and sequences utilised, for the included studies. The number of studies that reported differently the abnormality appearance on the same disease and using the same MR method specified (i.e. contradictory results) is also recorded.

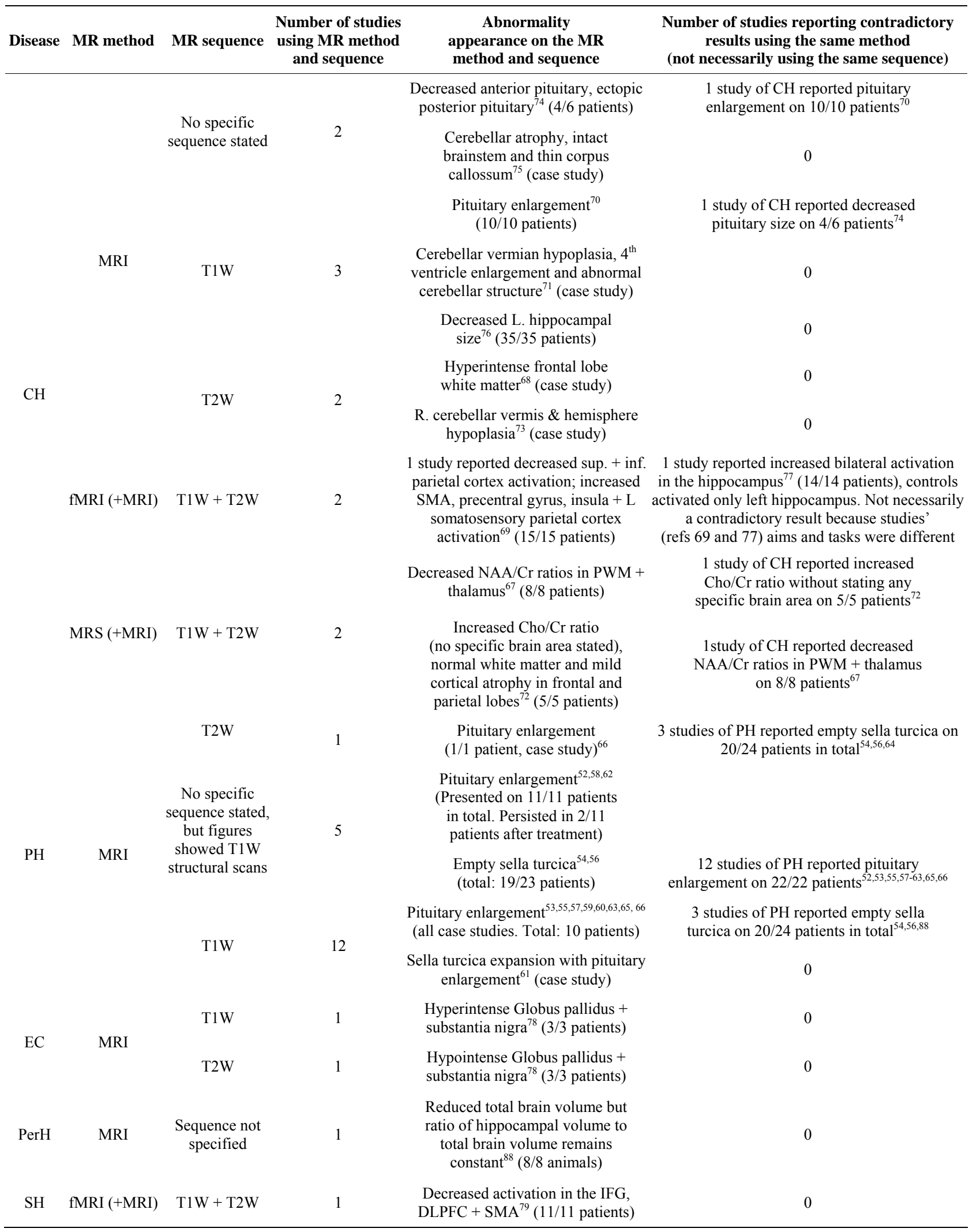


Two studies found abnormalities in the hippocampus [76,80]: Wheeler et al. [76] found that the left hippocampus specifically was reduced in size in 35 patients with congenital hypothyroidism, where as Hasegawa et al. [80] found that, although the hippocampal volume was decreased in mammals with perinatal hypothyroidism, this reduction did not affect the hippocampal to brain volume ratio and so was merely part of generalised brain atrophy. One fMRI study found increased bilateral activation in the hippocampus of 14/14 congenital hypothyroid patients, in contrast with $15 / 15$ controls in which increased activation was only observed in the left hippocampus [77] during a visuospatial memory task.

Tai et al. [78] found that three ID patients all showed Sylvian fissure enlargement and abnormal signal intensity in the globus pallidus and substantia nigra on different MRI methods (i.e. hyperintense on T1W and hypointense on $\mathrm{T} 2 \mathrm{~W}$ ). These structures are very metabolically active and contain large amounts of iodine [83] and so may be more susceptible to insults caused by ID. Additionally, Alves et al. [68] found hyperintense white matter in the anterior and posterior forceps, splenium and frontal lobes on T2W MRI.

The two MRS studies included obtained different results despite studying the same disease: one found increased $\mathrm{Cho} / \mathrm{Cr}$ ratio and no change in the $\mathrm{NAA} / \mathrm{Cr}$ ratio but did not specify the brain area affected [72]; the other discovered decreased NAA/Cr ratios in the parietal white matter and thalamus and no $\mathrm{Cho} / \mathrm{Cr}$ ratio abnormalities [67].

Blasi et al. [69] used fMRI to show that 15 congenital hypothyroid patients had greater activation in the bilateral supplementary motor area, the opercular area of the precentral gyrus, the left somatosensory parietal cortex and the corresponding insula whilst control subjects had greater activation in the superior parietal cortex when performing a mental rotation task. Congenital hypothyroid patients also deactivated the inferior parietal cortex to a greater extent than controls. Zhu et al. [79] used fMRI analysis on 11 patients and found that euthyroid patients activated five regions of interest when performing the task but that subclinical hypothyroid subjects only activated $2 / 5$ of these regions (bilateral parietal areas and bilateral premotor areas) [79]. The blood oxygen level dependant response was not seen in the middle/inferior frontal gyri, bilateral dorsolateral prefrontal cortex or supplementary motor area/anterior cingulate cortex suggesting that the frontal cortex is thus an area of abnormality and that working memory may be affected in iodine deficient subjects [79].

\subsection{Geographical Area of the Population Studied in Different Studies}

Studies from 10 countries contributed data to this review: India (6 studies), USA (6 studies), China (4 studies), Italy and Japan (Hokkaido prefecture) (3 studies each), Canada (2 studies) and The Netherlands, Spain, Russia and Turkey (1 study each) (Figure 4). From these countries, only Italy and Russia are known to have mild to moderate iodine deficiency in 2011 [84] (based on median urinary iodine level: UIC from 20 to $99 \mu \mathrm{g} / \mathrm{L}$ ).

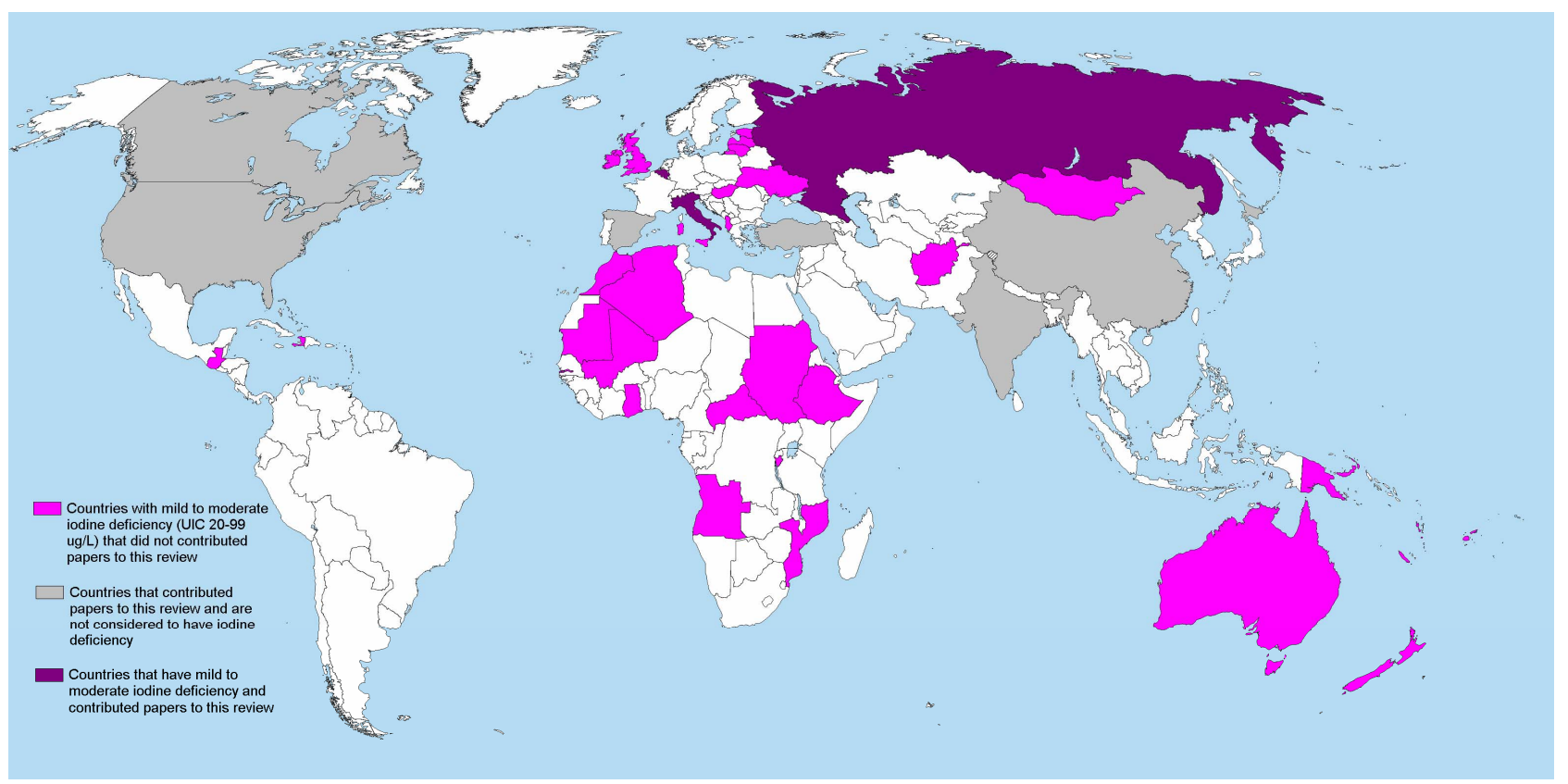

Figure 4. Map showing the countries that have mild to moderate iodine deficiency based on median urinary iodine concentration (UIC) as reported in 2011 by Anderssson et al., 2012 [84] and those from which data contributed to this review. 
However, publications from India, Turkey and China refer ID as a health issue in some regions. In terms of sample size, the majority of the data analysed by this review corresponds to studies from Canada (not considered to have ID) and from the northern region of Japan: known as having high iodine intake (UIC more than 300 $\mu \mathrm{g} / \mathrm{L}$ [84]), on a study that screened 83232 neonates, reported symptoms of ID related disease in 47 and confirmed it in 6 [74].

\section{Discussion}

Iodine deficiency results in metabolic and/or structural brain alterations which are detectable on MR imaging techniques. Of the papers identified as potentially relevant, only 29/82 confirmed the presence of the brain changes by applying MR techniques, as the majority of studies in this field rely on histochemical findings as depicting structural brain changes. Six studies on hypothyroidism (ID related disorder) were excluded as ID was not explicitly stated as the cause of the disease [Exclusion criteria]. However, 3 of them use, in addition to MRI, a nuclear medical imaging technique: Positron Emission Tomography (PET) $[85,86]$ and Single Photon Emission Computed Tomography (SPECT) [87]. This indicates that the spectrum of medical imaging techniques applied to study brain changes associated with ID related disorders is wide.

Although thyroid disorders related to ID can produce identifiable structural brain abnormalities, these can be highly inconsistent (Table 3). Despite the prevalence of pituitary abnormalities (identified by 17/29 studies), this finding was highly contradictory (enlargement was reported in 32 subjects whilst size reduction or empty sella in 30). Cerebellar and hippocampal abnormalities were, however, consistently reported throughout few of the studies included, acknowledged by others [6,7] and found in animals $[29,30,40,80]$. Nevertheless, the majority of the studies included in this review overlooks basic structural brain changes (e.g. global or regional atrophy) and were limited to visual regional descriptions. MRI was used in most of the studies included in this review; which was dominated by research in the context of primary and congenital hypothyroidism, both in terms of the number of studies and the subject sample size.

Despite the recognized impact of ID in human health, the low prevalence of ID disorders in the general population may have incidence in the lack of large-sized studies evaluating structural brain changes in this condition. The median sample size was 5 , as many of the included studies are single case reports. Including studies which encompassed iodine deficiency disorders rather than ID alone would have increased the scope of the review, but could also have meant that other non-iodine related consequences of these diseases may have affected the ap- pearance of the brain changes. Several of the case studies also often presented no methods sections and so MRI acquisition methods and statistical analysis methods may have been inadequate. With little evidence of statistical analysis, the significance of structural brain abnormalities/changes may have been unreliable; these brain structure sizes could actually be considered within the normal range if not compared with acceptable control brain scans. Additionally, representative images were frequently presented in these case studies, increasing selection bias since researchers were not blinded to case/ control status.

Blasi et al. [69] and Wheeler et al. [76] were the only included studies from which utilised researcher blinding. However, these studies are inclined to create other assumptions. Blasi et al. [69] uses the siblings of the congenital hypothyroid patients as control subjects, who may also have been affected by iodine ID in the environment and so may be predisposed to brain changes that have not yet manifested clinically or are not yet visible on MRI. Blasi et al. [69] additionally used fMRI along with a mental rotation task. The differences in blood oxygen level dependant response activation between cases and controls may be due to hypothyroid patients disengaging from the task rather than activating different, resultant brain areas [88] and there is also no guarantee in fMRI that subjects are performing the task as intended rather than thinking of other, irrelevant information, thus activating non-task dependent brain areas. Wheeler et al. [76] stated that the MRI data acquired from 'unacceptable' scans was disregarded and the measurements from subjects in the same group and of the same age and gender were used instead. The researchers did not clarify how many scans were deemed inappropriate and they did not provide brain scan exclusion criteria specifically and so again, selection bias is prevalent. It also assumes that the disregarded scans would have been similar to those of the other subjects.

Many studies were conducted to ascertain the effects of levothyroxine therapy in hypothyroid patients (i.e. whether brain abnormalities seen on MR could be reversed with this treatment). Since these are not directly related to the effects of ID, dependability becomes more disputable. Other sources of inconsistency include the use of differing field strengths among several studies (ranging from $0.2 \mathrm{~T}$ to $7 \mathrm{~T}$ ) and specific MR sequences are also not always provided. Pituitary hyperplasia is often confused with pituitary adenomas $[55,62]$. Hence, a consistent field strength which adequately displays these differences on MR images is necessary. In the study by Akinci et al. [67], researchers performed a whole body MRI scan; the clarification of small brain structure details may have been improved by using a head coil. Several studies also did not detail the time-frame between 
repeat MRI scans and so differences between studies in terms of time elapsed could increase result discrepancies.

Tai et al. [78] discuss the brain changes found as being in the areas of the globus pallidus and substantia nigra; hyperintense on T1W and hypointense on T2W. However, no distinction is given as to the degree of hyper- or hypo-intensity for which an appropriate grading scale consistent across all studies is required. The research in this paper could also have been improved by the additional utilisation of Computed Tomography (CT) scans in all subjects; this would enable authors to determine whether the change in intensity was due to calcifications and thus prevent assumptions.

To the best of our knowledge, there has not been a systematic review before on the appearance of brain changes on MR caused by iodine deficiency. Despite the large worldwide availability of MR techniques and the importance of the study of iodine insufficiency, given its strong link with neurodevelopment and impaired cognition, most current literature on ID reports histological and cytoarchitectural brain alterations only, with no MR confirmation. In some countries known as having mild to moderate ID the major causes of the disorders included in this review are immunological or iatrogenic rather than ID (e.g. United Kigdom). Therefore this review, rather than reflecting the spectrum of ID related brain abnormalities provides guidance and constitutes a call for future studies in ID and brain health.

Strengths of this work include searching multiple databases over a large time-frame, the use of comprehendsive search terms, the inclusion of fMRI and MRS studies, the inclusion of ID diseases rather than research discussing ID alone, the inclusion of both human and animal studies and the inclusion of healthy and diseased brains. A weakness, however, is the exclusion of nonEnglish papers.

The literature on brain structural and metabolic abnormalities related to ID that are documented using MR methods is limited to few studies with differing field strengths and MR sequences, small sample sizes and poor evidence of blinding, randomisation and an inclusion/exclusion criteria in many of them. Interobserver bias also affects some of the included studies; for example, in the study by Hasegawa et al. [80] (the only included study that uses randomisation) volumetric analysis of the hippocampus was manually performed by one researcher only. Additionally, the limited description of the signal changes (when mentioned) lacks from an adequate (i.e. normalised) grading, all of which attempts against the scope of the results obtained.

\section{Directions for Future Research}

More studies are required which correlate histological findings with MR image results and the utilisation of more standardised sequences and field strengths are necessary. With the increased focus on the consequence of iodine insufficiency both in utero and in infancy, it is important to understand the brain structural modifications caused by ID, given that neurodevelopmental and cognitive outcomes range in severity according to the level and timing of ID. Future work should include global and regional atrophy changes to identify directions for the research that will improve the understanding of the pathophysiological mechanisms related with the effects of ID in the brain.

\section{Acknowledgements}

This work was mainly funded by the College of Medicine and Veterinary Medicine of the University of Edinburgh, with partial funds from Row Fogo Charitable Trust and SINAPSE (Scottish Imaging Network A Platform for Scientific Excellence) collaboration. Funds from the Royal Society of Edinburgh, NESTA (National Endowment for Science, Technology and the Arts) and The Scottish Funding Council through the Scottish Crucible initiative are also gratefully acknowledged.

\section{REFERENCES}

[1] B. But, C. W. Chan, F. Chan, K. W. Chan, A. W. Cheng, P. Cheung, et al., "Consensus Statement on Iodine Deficiency Disorders in Hong Kong," Hong Kong Medical Journal, Vol. 9, No. 6, 2003, pp. 446-453.

[2] V. Sethi and U. Kapil, "Iodine Deficiency and Development of the Brain," Indian Journal of Pediatrics, Vol. 71, No. 4, 2004, pp. 325-329.

http://dx.doi.org/10.1007/BF02724099

[3] D. V. Becker, L. E. Braverman, F. Delange, J. T. Dunn, J. A. Franklyn, J. G. Hollowell, et al., "Iodine Supplementation for Pregnancy and Lactation-United States and Canada: Recommendations of the American Thyroid Association: The Public Health Committee of the American Thyroid Association," Thyroid, Vol. 16, No. 10, 2006, pp. 949-951. http://dx.doi.org/10.1089/thy.2006.16.949

[4] M. D. H. Ibrahim, J. K. H. Sinn and W. McGuire, "Iodine Supplementation for the Prevention of Mortality and Adverse Neurodevelopmental Outcomes in Preterm Infants," Cochrane Database of Systematic Reviews, Vol. 2, 2006, pp. 1-18. http://dx.doi.org/10.1002/14651858.CD005253

[5] J. P. Schroder-van der Elst, D. Van der Heide, G. M. de Escobar and M. J. Obregon, "Iodothyronine Deiodinase Activities in Fetal Rat Tissues at Several Levels of Iodine Deficiency: A Role for the Skin in 3,5,3-Triiodothyronine Economy?" Endocrinology, Vol. 139, No. 5, 1998, pp. 2229-2234. http://dx.doi.org/10.1210/en.139.5.2229

[6] F. Delange, "The Role of Iodine in Brain Development," Proceedings of the Nutrition Society, Vol. 59, No. 1, 2000, pp. 75-79. http://dx.doi.org/10.1017/S0029665100000094

[7] Delange F, "Iodine Deficiency as a Cause of Brain Damage," Postgraduate Medical Journal, Vol. 77, No. 906, 
2001, pp. 217-220.

http://dx.doi.org/10.1136/pmj.77.906.217

[8] F. Delange, "Epidemiology and Impact of Iodine Deficiency in Pediatrics," Journal of Pediatric Endocrinology \& Metabolism, Vol. 18, Suppl. 1, 2005, pp. 1245-1251. http://dx.doi.org/10.1515/JPEM.2005.18.S1.1245

[9] M. P. J. Vanderpump, J. H. Lazarus, P. P. Smith, P. Laurberg, R. L. Holder, K. Boelaert, et al., "Iodine Status of UK Schoolgirls: A Cross-Sectional Survey," Lancet, Vol. 377, No. 9782, 2011, pp. 2007-2012. http://dx.doi.org/10.1016/S0140-6736(11)60693-4

[10] R. Lavado-Autric, E. Auso, J. V. Garcia-Velasco, M. del Carmen Arufe, F. E. del Rey, P. Berbel, et al., "Early Maternal Hypothyroxinemia Alters Histogenesis and Cerebral Cortex Cytoarchitecture of the Progeny," Journal of Clinical Investigation, Vol. 111, No. 7, 2003, pp. 10731082. http://dx.doi.org/10.1172/JCI200316262

[11] N. Van Wijk, E. Rijntjes and B. J. M. van de Heijning, "Perinatal and Chronic Hypothyroidism Impair Behavioural Development in Male and Female Rats," Experimental Physiology, Vol. 93, No. 11, 2008, pp. 1199-1209. http://dx.doi.org/10.1113/expphysiol.2008.042416

[12] F. R. S. Lima, A. Gervais, C. Colin, M. Izembart, V. M. Neto and M. Mallat, "Regulation of Microglial Development: A Novel Role for Thyroid Hormone," Journal of Neuroscience, Vol. 21, No. 11, 2001, pp. 2028-2038. http://dx.doi.org/10.1113/expphysiol.2008.042416

[13] A. R. Mansourian, "A Review on the Metabolic Disorders of Iodine Deficiency," Pakistan Journal of Biological Sciences, Vol. 14, No. 7, 2011, pp. 412-424. http://dx.doi.org/10.3923/pjbs.2011.412.424

[14] N. Bleichrodt and M. P. Born, "Meta-Analysis of Research on Iodine and Its Relationship to Cognitive Development," In: J. B. Stanbury, Ed., The Damaged Brain of Iodine Deficiency, Cognizant Communication Corporation, New York, 1994, pp. 195-200.

[15] D. Glinoer, "The Importance of Iodine Nutrition during Pregnancy," Public Health Nutrition, Vol. 10, No. 12A, 2007, pp. 1542-1546.

http://dx.doi.org/10.1017/S1368980007360886

[16] C. Mian, P. Vitaliano, D. Pozza, S. Barollo, M. Pitton, G. Callegari, et al., "Iodine Status in Pregnancy: Role of Dietary Habits and Geographical Origin," Clinical Endocrinology, Vol. 70, No. 5, 2009, pp. 776-780. http://dx.doi.org/10.1111/j.1365-2265.2008.03416.x

[17] M. B. Zimmermann, "Iodine Deficiency in Pregnancy and the Effects of Maternal Iodine Supplementation on the Offspring: A Review", American Journal of Clinical Nutrition, Vol. 89, No. 2, 2009, pp. 668S-672S. http://dx.doi.org/10.3945/ajcn.2008.26811C

[18] D. Fuehrer, “Thyroid Illness during Pregnancy," Internist (Berl), Vol. 52, 2011, pp. 1158-1166.

[19] C. A. Barnett, T. J. Visser, F. Williams, H. V. Toor, S. Duran, M. J. Presas, et al., "Inadequate Iodine Intake of $40 \%$ of Pregnant Women from a Region in Scotland", Journal of Endocrinological Investigation, Vol. 25, 2002, p. 90 .

[20] D. Glinoer, "The Regulation of Thyroid Function during
Normal pregnancy: Importance of the Iodine Nutrition Status," Best Practice \& Research Clinical Endocrinology \& Metabolism, Vol. 18, No. 2, 2004, pp. 133-152. http://dx.doi.org/10.1016/j.beem.2004.03.001

[21] F. R. Perez-Lopez, "Iodine and Thyroid Hormones during Pregnancy and Postpartum," Gynecological Endocrinology, Vol. 23, No. 7, 2007, pp. 414-428. http://dx.doi.org/10.1080/09513590701464092

[22] N. Kochupillai and M. Mehta, "Iodine Deficiency Disorders and Their Prevention in India," Reviews of Endocrinology and Metabolic Disorders, Vol. 9, No. 3, 2008, pp. 237-244. http://dx.doi.org/10.1007/s11154-008-9094-0

[23] A. Melse-Boonstra, N. Jaiswal, "Iodine Deficiency in Pregnancy, Infancy and Childhood and its Consequences for Brain Development," Best Practice \& Research Clinical Endocrinology \& Metabolism, Vol. 24, No. 1, 2010, pp. 29-38. http://dx.doi.org/10.1016/j.beem.2009.09.002

[24] J. H. Lazarus, "Evaluating Iodine Deficiency in Pregnant Women and Young Infants-Complex Physiology with a Risk of Misinterpretation-Comments," Public Health Nutrition, Vol. 10, No. 12A, 2007, pp. 1553-1553.

[25] M. Haldimann, A. Alt, A. Blanc and K. Blondeau, "Iodine Content of Food Groups", Journal of Food Composition and Analysis, Vol. 18, No. 6, 2005, pp. 461-471. http://dx.doi.org/10.1016/j.jfca.2004.06.003

[26] G. M. De Escobar, M. J. Obregon and F. E. del Rey, "Iodine Deficiency and Brain Development in the First Half of Pregnancy," Public Health Nutrition, Vol. 10, No. 12A, 2007, pp. 1554-1570.

http://dx.doi.org/10.1017/S1368980007360928

[27] D. Benton, "The Influence of Dietary Status on the Cognitive Performance of Children," Molecular Nutrition \& Food Research, Vol. 54, No. 4, 2010, pp. 457-470. http://dx.doi.org/10.1002/mnfr.200900158

[28] B. S. Hetzel, "Iodine and Neuropsychological Development," Journal of Nutrition, Vol. 130, 2000, pp. 493S495S.

[29] B. J. Potter, M. T. Mano, G. B. Belling, D. M. Martin, B. G. Craqq, J. Chavadej, et al., "Restoration of Brain Growth in Fetal Sheep after Iodized Oil Administration to IodineDeficient Ewes", Journal of Neurological Sciences, Vol. 66, 1984, pp. 15-26. http://dx.doi.org/10.1016/0022-510X(84)90137-0

[30] A. E. Voudouri, E. C. Stella, J. G. Menegatos, G. P. Zervas, F. Nicol and J. R. Arthur, "Selenoenzyme Activities in Selenium and Iodine-Deficient Sheep," Biological Trace Element Research, Vol. 94, No. 3, 2003, pp. 213 224. http://dx.doi.org/10.1385/BTER:94:3:213

[31] A. Farahvar, N. H. Darwish, S. Sladek and E. Meisami, "Marked Recovery of Functional Metabolic Activity and Laminar Volumes in the Rat Hippocampus and Dentate Gyrus Following Postnatal Hypothyroid Growth Retardation: A Quantitative Cytochrome Oxidase Study," Experimental Neurology, Vol. 204, No. 2, 2007, pp. 556-568. http://dx.doi.org/10.1016/j.expneurol.2006.12.019

[32] B. Giray, J. Riondel, J. Arnaud, V. Ducros, A. Favier and F. Hincal, "Iodine and/or Selenium Deficiency Alters Tissue Distribution Pattern of Other Trace Elements in 
Rats," Biological Trace Element Research, Vol. 95, No. 3, 2003, pp. 247-258.

http://dx.doi.org/10.1385/BTER:95:3:247

[33] N. Q. Liu, Q. Xu, X. L. Hou, P. S. Liu, Z. F. Chai, L. Zhu, et al., "The Distribution Patterns of Trace Elements in the Brain and Erythrocytes in a Rat Experimental Model of Iodine Deficiency," Brain Research Bulletin, Vol. 15, No. 2, 2001, pp. 309-312. http://dx.doi.org/10.1016/S0361-9230(01)00508-1

[34] F. Zhang, N. Liu, X. Zhao, A. Zuo, L. Yang, Q. Xu, et al., "Variations of Elemental Distribution in Brain Regions of Neonatal Rats at Different Iodine Intakes," Biological Trace Element Research, Vol. 90, No. 1-3, 2002, pp. 227-237. http://dx.doi.org/10.1385/BTER:90:1-3:227

[35] Y. Ge, R. Niu, J. Zhang and J. Wang, "Proteomic Analysis of Brain Proteins of Rats Exposed to High Xuoride and Low Iodine," Archives of Toxicology, Vol. 85, No. 1, 2011, pp. 27-33.

http://dx.doi.org/10.1007/s00204-010-0537-5

[36] A. G. Ferraz, F. E. del Rey, G. M. de Escobar, G. M. Innocenti and P. Berbel, "The Development of the Anterior Commissure in Normal and Hypothyroid Rats", Developmental Brain Research, Vol. 81, No. 2, 1994, pp. 293-308. http://dx.doi.org/10.1016/0165-3806(94)90315-8

[37] J. H. Mitchell, F. Nicol, G. J. Beckett and J. R. Arthur, "Selenoprotein Expression and Brain Development in Preweanling Selenium- and Iodine-Deficient Rats", Journal of Molecular Endocrinology, Vol. 20, 1998, pp. 203210. http://dx.doi.org/10.1677/jme.0.0200203

[38] Y. Ge, H. Ning, S. Wang and J. Wang, "Effects of High Fluoride and Low Iodine on Brain Histopathology in Offspring Rats," Fluoride, Vol. 38, 2005, pp. 127-132.

[39] Y. Ge, H. Ning, C. Feng, H. Wang, X. Yan, S. Wang, et al., "Apoptosis in Brain Cells of Offspring Rats Exposed to High Fluoride and Low Iodine," Fluoride, Vol. 39, 2006, pp. 173-178.

[40] G. H. McIntosh, D. A. Howard, M. T. Mano, M. L. Wellby and B. S. Hetzel, "Iodine Deficiency and Brain Development in the Rat," Australian Journal of Biological Sciences, Vol. 34, 1981, pp. 427-433.

[41] M. Hadjzadeh, A. K. Sinha, M. R. Pickard and R. P. Ekins, "Effect of Maternal Hypothyroxinaemia in the Rat on Brain Biochemistry in Adult Progeny," Journal of Endocrinology, Vol. 124, 1990, pp. 387-396. http://dx.doi.org/10.1677/joe.0.1240387

[42] V. J. Pop, E. P. Brouwers, H. L. Vader, T. Vulsma, A. L. van Baar and J. J. de Vijlder, "Maternal Hypothyroxinaemia during Early Pregnancy and Subsequent Child Development: A 3-Year Follow-Up Study," Clinical Endocrinology, Vol. 59, 2003, pp. 282-288.

[43] R. P. Rooman, M. V. L. Du Caju, L. Op De Beeck, M. Docx, P. Van Reempts and K. J. Van Acker, "Low Thyroxinaemia Occurs in the Majority of Preterm Newborns," European Journal of Pediatrics, Vol. 155, No. 3, 1996, pp. 211-215.

http://dx.doi.org/10.1007/BF01953940

[44] O. P. Soldin, "Hypothyroxinaemia, Iodine Deficiency, and Subtle Changes in Migration and Cytoarchitecture," Environmental Health Perspectives, Vol. 112, 2004, pp. A268-A269. http://dx.doi.org/10.1289/ehp.112-a268c

[45] G. M. De Escobar, M. J. Obregon and F. E. del Rey, "Role of Thyroid Hormone during Early Brain Development," European Journal of Endocrinology, Vol. 151, 2004, pp. U25-U37. http://dx.doi.org/10.1530/eje.0.151U025

[46] F. Williams and R. Hume, "The Measurement, Definition, Aetiology and Clinical Consequences of Neonatal Transient Hypothyroxinaemia," Annals of Clinical Biochemistry, Vol. 48, No. 1, 2011, pp. 7-22. http://dx.doi.org/10.1258/acb.2010.010174

[47] K. Beardsall and A. L. Ogilvy-Stuart, "Congenital Hypothyroidism," Current Paediatrics, Vol. 14, No. 5, 2004, pp. 422-429. http://dx.doi.org/10.1016/j.cupe.2004.05.006

[48] G. Cleghorn, "The Role of Red Meat in the Diet for Children and Adolescents," Nutrition \& Dietetics, Vol. 64, Suppl. 4, 2007, pp. S143-S146.

http://dx.doi.org/10.1111/j.1747-0080.2007.00203.x

[49] J. Bernal, "Iodine and Brain Development," BioFactors, Vol. 10, No. 2-3, 1999, pp. 271-276. http://dx.doi.org/10.1002/biof.5520100227

[50] J. P. Halpern, S. C. Boyages, G. F. Maberly, J. K. Collins, C. J. Eastman, J. G. L. Morris. "The Neurology of Endemic Cretinism: A Study of Two Endemias," Brain, Vol. 114, 1991, pp. 825-841. http://dx.doi.org/10.1093/brain/114.2.825

[51] N. Kretchmer, J. L. Beard and S. Carlson, "The Role of Nutrition in the Development of Normal Cognition," American Journal of Clinical Nutrition, Vol. 63, No. 6, 1996, pp. 997S-1001S.

[52] X. Aijing and L. Tang, "Pituitary Hyperplasia in Children with Short Stature and Primary Hypothyroidism," Indian Pediatrics, Vol. 47, No. 10, 2010, pp. 877-880. http://dx.doi.org/10.1007/s13312-010-0149-4

[53] W. W. Ashley, J. G. Ojemann, T. S. Park, M. D. Wippold, "Primary Hypothyroidism in a 12-Year Old Girl with a Suprasellar Pituitary Mass: Rapid Regression After Thyroid Replacement Therapy-Case Report," Journal of Neurosurgery, Vol. 102, No. 4, 2005, pp. 0413-416.

[54] I. I. Dedov, T. S. Zenkova, G. A. Mel'nichenko, O. I. Belichenko and I. D. Fedina, "The Potentials of Magnetic Resonance Tomography in the Diagnosis of the 'Empty' Sella Turcica," Neuroscience and Behavioural Physiology, Vol. 24, No. 3, 1994, pp. 229-233. http://dx.doi.org/10.1007/BF02362026

[55] P. U. Ehirim, D. S. Kerr and A. R. Cohen, "Primary Hypothyroidism Mimicking a Pituitary Macroadenoma," Pediatric Neurosurgery, Vol. 28, No. 4, 1998, pp. 195197. http://dx.doi.org/10.1159/000028649

[56] R. Garcia-Centeno, J. P. Suarez-Llanos, E. FernandezFernandez, V. Andia-Melero, P. Sanchez and A. JaraAlbarran, "Empty Sella and Primary Autoimmune Hypothyroidism," Clinical and Experimental Medicine, Vol. 10, No. 2, 2010, pp. 129-134. http://dx.doi.org/10.1007/s10238-009-0071-z

[57] R. Goswami, N. Tandon, R. Sharma and N. Kochupillai, 
"Residual Pituitary Enlargement in Primary Hypothyroidism Despite 1 1/2 Years of L-Thyroxine Therapy," Australasian Radiology, Vol. 43, No. 1, 1999, pp. 121123. http://dx.doi.org/10.1046/j.1440-1673.1999.00610.x

[58] J. M. Kroese, A. F. Grootendorst and L. J. D. M. Schelfhout, "Postpartum Amenorrhoea-Galactorrhoea Associated with Hyperprolactinaemia and Pituitary Enlargement in Primary Hypothyroidism," The Netherlands Journal of Medicine, Vol. 62, No. 1, 2004, pp. 28-30.

[59] C. Y. Lee, H. H. Hsu, H. Y. Lai and S. T. Lee, "Rapid Progression of Hypothyroidism-Related Pituitary Hyperplasia," Journal of Neurosurgery Pediatrics, Vol. 2, No. 3, 2008, pp. 212-214.

http://dx.doi.org/10.3171/PED/2008/2/9/212

[60] E. Passeri, A. Tufano, M. Locatelli, A. G. Lania, B. Ambrosi and S. Corbetta, "Large Pituitary Hyperplasia in Severe Primary Hypothyroidism," Journal of Clinical Endocrinology and Metabolism, Vol. 96, No. 1, 2011, pp. 22-23. http://dx.doi.org/10.1210/jc.2010-2011

[61] P. J. Shogan and M. Monson, "Enlarged Sella of Primary Childhood Hypothyroidism," Pediatric Radiology, Vol. 40, Suppl. 1, 2010, p. S163.

http://dx.doi.org/10.1007/s00247-010-1561-6

[62] M. Young, K. Kattner and K. Gupta, "Pituitary Hyperplasia Resulting from Primary Hypothyroidism Mimicking Macroadenomas," British Journal of Neurosurgery, Vol. 13, No. 2, 1999, pp. 138-142. http://dx.doi.org/10.1080/02688699943880

[63] N. Sengupta, U. Sinha, K. Sinha Roy and S. Saha, "Acromegaly without Acral Changes: A Rare Presentation", Indian Journal of Endocrinology and Metabolism, Vol. 16, No. 3, 2012, pp. 457-459. http://dx.doi.org/10.4103/2230-8210.95713

[64] D. Dutta, I. Maisnam, S. Ghosh, P. Mukhopadhyay, S. Mukhopadhyay and S. Chowdhury, "Panhypopituitarism with Empty Sella a Sequel of Pituitary Hyperplasia Due to Chronic Primary Hypothyroidism," Indian Journal of Endocrinology and Metabolism, Vol. 16, 2012, pp. S282S284.

[65] J. A. Atchison, P. A. Lee and A. L. Albright, "Reversible Suprasellar Pituitary Mass Secondary to Hypothyroidism," JAMA, Vol. 262, No. 22, 1989, pp. 3175-3177. http://dx.doi.org/10.1001/jama.1989.03430220098038

[66] A. Agrawal and S. K. Diwan, "Pituitary Hyperplasia Resulting from Primary Hypothyroidism," Asian Journal of Neurosurgery, Vol. 6, No. 2, 2011, pp. 99-100. http://dx.doi.org/10.4103/1793-5482.92171

[67] A. Akinci, K. Sarac, S. Gungor, I. Mungan and O. Aydin, "Brain MR Spectroscopy Findings in Neonates with Hypothyroidism Born to Mothers Living in Iodine-Deficient Areas," American Journal of Neuroradiology, Vol. 27, No. 10, 2006, pp. 2083-2087.

[68] C. Alves, M. Eldson, H. Engle, J. Sheldon and W. W. Cleveland, "Changes in Brain Maturation Detected by Magnetic Resonance Imaging in Congenital Hypothyroidism," Journal of Pediatrics, Vol. 115, No. 4, 1989, pp. 600-603.

http://dx.doi.org/10.1016/S0022-3476(89)80292-6
[69] V. Blasi, R. Longaretta, C. Giovanettoni, C. Balodi, S. Pontesilli, C. Vigone, et al., "Decreased Parietal Cortex Activity during Mental Rotation in Children with Congenital Hypothyroidism," Neuroendocrinology, Vol. 89, No. 4, 2009, pp. 56-65. http://dx.doi.org/10.1159/000151397

[70] M. P. Desai, R. U. Mehta, C. S. Choksi and M. P. Colaco, "Pituitary Enlargement on Magnetic Resonance Imaging in Congenital Hypothyroidism," Archives of Pediatrics and Adolescent Medicine, Vol. 150, No. 6, 1996, pp. 623628.

http://dx.doi.org/10.1001/archpedi.1996.02170310057010

[71] J. J. Graber, H. Lau and S. Sathe, "Teaching Neuroimages: Molar Tooth Sign with Hypotonia, Ataxia, and Nystagmus (Joubert Syndrome) and Hypothyroidism," Neurology, Vol. 73, No. 24, 2009, p. e106.

http://dx.doi.org/10.1212/WNL.0b013e3181c679ba

[72] R. K. Gupta, V. Bhatia, H. Poptani and R. B. Gujral, "Brain Metabolite Changes on In Vivo Proton Magnetic Resonance Spectroscopy in Children with Congenital Hypothyroidism," Journal of Pediatrics, Vol. 126, No. 3, 1995, pp. 389-392. http://dx.doi.org/10.1016/S0022-3476(95)70454-X

[73] L. Mauceri, M. Ruggieri, V. Pavone, R. Rizzo and G. Sorge, "Craniofacial Anomalies, Severe Cerebellar Hypoplasia, Psychomotor and Growth Delay in a Child with Congenital Hypothyroidism," Clinical Dysmorphology, Vol. 6, No. 4, 1997, pp. 375-378. http://dx.doi.org/10.1097/00019605-199710000-00013

[74] F. Fujiwara, K. Fujikura, K. Okuhara, J. Tsubaki, M. Fukushi, K. Fujita, et al., "Central Congenital Hypothyroidism Detected by Neonatal Screening in Sapporo, Japan (2000-2004): It's Prevalence and Clinical Characteristics," Clinical Pediatric Endocrinology, Vol. 17, No. 3, 2000, pp. 65-69. http://dx.doi.org/10.1297/cpe.17.65

[75] T. Tajima, F. Fujiwara, A. Sudo, S. Saito and K. Fujieda, "A Japanese Patient of Congenital Hypothyroidism with Cerebellar Atrophy," Endocrine Journal, Vol. 54, No. 6, 2007, pp. 941-944.

http://dx.doi.org/10.1507/endocrj.K07-105

[76] S. M. Wheeler, K. A. Willoughby, M. P. McAndrews and J. F. Rovet, "Hippocampal Size and Memory Functioning in Children and Adolescents with Congenital Hypothyroidism," Journal of Clinical Endocrinology \& Metabolism, Vol. 96, No. 9, 2011, pp. E1427-E1434. http://dx.doi.org/10.1210/jc.2011-0119

[77] S. M. Wheeler, M. P. McAndrews, E. D. Sheard and J. Rovet, "Visuospatial Associative Memory and Hippocampal Functioning in Congenital Hypothyroidism", Journal of the International Neuropsychological Society, Vol. 18, 2012, pp. 49-56. http://dx.doi.org/10.1017/S1355617711001378

[78] M. Tai, C. Lian, S. P. Qi, E. R. Heinz and G. R. DeLong, "Magnetic Resonance Imaging of Brain and the Neuromotor Disorder in Endemic Cretinism," Annals of Neurology, Vol. 34, No. 1, 1993, pp. 91-94. http://dx.doi.org/10.1002/ana.410340116

[79] D. F. Zhu, Z. X. Wang, D. R. Zhang, Z. L. Pan, S. He, X. P. Hu, et al., "FMRI Revealed Neural Substrate for Re- 
versible Working Memory Dysfunction in Subclinical Hypothyroidism," Brain, Vol. 129, No. 11, 2006, pp. 2923-2930. http://dx.doi.org/10.1093/brain/awl215

[80] M. Hasegawa, I. Kidac and H. Wadaa, “A Volumetric Analysis of the Brain and Hippocampus of Rats Rendered Perinatal Hypothyroid," Neuroscience Letters, Vol. 479, No. 3, 2010, pp. 240-244. http://dx.doi.org/10.1016/j.neulet.2010.05.070

[81] A. Liberati, D. G. Altman, J. Tetzlaff, C. Mulrow, P. C. Gøtzche, J. P. A. Ioannidis, et al., "The PRISMA Statement for Reporting Systematic Reviews and MetaAnalyses of Studies that Evaluate healthcare Interventions: Explanation and Elaborations", BMJ, Vol. 339, 2009, p. b2700. http://dx.doi.org/10.1136/bmj.b2700

[82] P. Whiting, A. W. S. Rutjes, J. B. Reitsma, P. M. M. Bossuyt and J. Kleijnen, "The development of QUADAS: A Tool for the Quality Assessment of Studies of Diagnostic Accuracy Included in Systematic Reviews," Medical Research Methodology, Vol. 3, 2003 p. 25. http://dx.doi.org/10.1186/1471-2288-3-25

[83] E. Andrasi, C. Belavari, V. Stibilj, M. Dermelj and D. Gawlik, "Iodine Concentration in Different Human Brain Parts," Analytical and Bioanalytical Chemistry, Vol. 378, No. 1, 2004, pp. 129-133. http://dx.doi.org/10.1007/s00216-003-2313-3

[84] M. Andersson, V. Karumbunathan and M. B. Zimmermann, "Global Iodine Status in 2011 and Trends over the
Past Decade”, Journal of Nutrition, Vol. 142, No. 4, 2012, pp. 744-750. http://dx.doi.org/10.3945/jn.111.149393

[85] M. Bauer, D.H. S. Silverman, F. Schlagenhauf, E. D. London, C. L. Geist, K. van Herle, et al., "Brain Glucose Metabolism in Hypothyroidism: A Possitron Emission Tomography Study before and after Thyroid Hormone Replacement Therapy," Journal of Clinical Endocrinology \& Metabolism, Vol. 94, No. 8, 2009, pp. 2922-2929. http://dx.doi.org/10.1210/jc.2008-2235

[86] E. L. Constant, A. G. De Volder, A. Ivanoiu, A. Bol, D. Labar, A. Seghers, et al., "Cerebral Blood Flow and Glucose Metabolism in Hypothyroidism: A Positron Emission Tomography Study," Journal of Clinical Endocrinology \& Metabolism, Vol. 86, No. 8, 2001, pp. 3864 3870.http://dx.doi.org/10.1210/jc.86.8.3864

[87] Y. Krausz, N. Freedman, H. Lester, J. P. Newman, G. Barkai, M. Bocher, et al., "Regional cerebral blood flow in patients with mild hypothyroidism," Journal of $\mathrm{Nu}$ clear Medicine, Vol. 545, 2004, pp. 1712-1715.

[88] J. H. Callicott, V. S. Mattay, A. Bertolino, K. Finn, R. Coppola, J. A. Frank, et al., "Physiological Characteristics of Capacity Constraints in Working Memory as Revealed by Functional MRI," Cerebral Cortex, Vol. 9, No. 1, 1999, pp. 20-26. http://dx.doi.org/10.1093/cercor/9.1.20

\section{Acronyms and Abbreviations for Tables}

PH: Primary Hypothyroidism;

$\mathrm{CH}$ : Congenital Hypothyroidism;

EC: Endemic Cretinism;

PerH: Perinatal Hypothyroidism;

SH: Subclinical Hypothyroidism;

$R$ : right; $L$ : left;

NAA: N-acetylaspartate;

Cr: Creatinine; Cho: Choline;

sup: Superior; inf: Inferior;

PWM Parietal White Matter;

IFG: Inferior Frontal Gyrus;

DLPFC: Dorsolateral Prefrontal Cortex;

SMA: Supplementary Motor Area;

$T 1 W$ : T1-weighted sequence;

T2W: T2-weighted sequence 